\title{
COMUNICAÇÃO PREJUDICADA: FATORES RELACIONADOS E CARACTERÍSTICAS DEFINIDORAS EM PACIENTES INTERNADOS COM QUEIMADURAS
}

\author{
Adriana Silva Meneguello * \\ Maria Célia Barcellos Dalri ** \\ Emília Campos de Carvalho *** \\ Maria Márcia Bachion ****
}

MENEGUELLO, A.S.; DALRI, M.C.B.; CARVALHO, E.C.de; BACHION, M.M. Comunicação prejudicada: fatores relacionados e características definidoras em pacientes internados com queimaduras. Rev.latino-am.enfermagem, Ribeirão Preto, v. 4, nº especial, p. 153- 72, abril 1996.

O presente estudo aborda as questões do paciente portador de queimaduras e suas influências no processo comunicacional. Busca contribuir com a preocupação recente da Enfermagem na criação de instrumentos de identificação de problemas de comunicação, a formulação de diagnósticos de enfermagem que envolve tais alterações, bem como, a elaboração de propostas alternativas para o cuidado a pacientes de comunicação prejudicada. A partir do conhecimento dos resultados de DALRI (1993), que encontrou 03 pacientes com diagnóstico de comunicação prejudicada, utilizando a Taxonomia I da North American Nursing Diagnosis Association (NANDA), realizamos uma análise secundária dos dados tomando como subsídio a proposta de CARVALHO \& COLER (1994). Nesta amostra, encontramos 14 pacientes com esta alternação (Comunicação Prejudicada), confirmando a hipótese de que a segunda proposta para o diagnóstico de enfermagem de Comunicação Prejudicada se apresenta mais abrangente. Os fatores relacionados mais freqüentes foram: condições físicas - alteração anatômica (aparelho visual e fonador); stress e percepção alterada. As características definidoras mais comuns foram: expressões de ansiedade/depressão; déficit visual parcial ou total e dispnéia.

UNITERMOS: diagnóstico de enfermagem; comunicação prejudicada.

\footnotetext{
* Aluna do $4^{\circ}$ ano de Graduação da Escola de Enfermagem de Ribeirão Preto da Universidade de São Paulo

** Diretora de Serviço do Centro Cirúrgico e Central de Material do Hospital das Clínicas da Faculdade de Medicina de Ribeirão Preto da Universidade de São Paulo - Unidade de Emergência

*** Professor Titular da Escola de Enfermagem de Ribeirão Preto da Universidade de São Paulo

**** Professor Assistente da Escola de Enfermagem de Ribeirão Preto da Universidade de São Paulo
}

Rev. Latino-am.enfermagem - Ribeirão Preto - v. 4 - n. especial - p. 153-172 - abril 1996153 


\section{INTRODUÇÃO}

$\mathrm{Na}$ enfermagem o processo de comunicação humana é de extrema relevância nos vários contextos do exercício profissional. Na relação diádica enfermeiro-paciente, em cujo contexto transcorre predominantemente a coleta de informações sobre o paciente, deve se considerar tanto os elementos inerentes ao profissional, como àqueles relativos ao paciente, ou seja: características genéticas, experiências culturais, crenças, valores e atitudes, nível de autoestima, características de personalidade e mecanismos de defesa utilizados (MCFARLAND \& NASCHINSKI, 1985). Temos ainda que considerar, segundo DALRI (1993), a idade, o desenvolvimento do ciclo vital, o sexo, a língua dominante, as barreiras psicológicas ou fisiológicas e os medicamentos, e a tolerância e atitude do paciente. Estes aspectos são relevantes para qualquer tipo de paciente, inclusive os que possuem trauma térmico; nestes casos alguns aspectos clínicos são mais intervenientes na comunicação com o paciente. Embora autores como LYNCH \& LEWIS (1973); ARTZ, JR. et al (1980); SMELTZER \& BARE (1994); GOMES et al (1995) tratem dos aspectos hemodinâmicos, gerais, de pacientes com trauma térmico, a literatura não evidencia a correspondência direta destes aspectos aos distúrbios de comunicação.

Destacamos, dentre estes, a alteração das percepções auditiva e visual, que poderá ser parcial ou total. Podemos exemplificar com o trauma térmico de face, em especial dos pavilhões auriculares, principalmente em decorrência de líquidos superaquecidos (óleo quente, leite quente, água fervendo) ou substâncias químicas, no qual o paciente manifestará impedimento ou diminuição no processo de recepção da comunicação. Já a alteração visual, assim como da fala podem ocorrer em decorrência a edemas severos.

Nestes casos o paciente desenvolve edema significativo, de grande magnitude, após 12 a 24 horas, em decorrência da frouxidão da pele facial e do seu rico suprimento sanguíneo. Este edema contribui para uma distorção anatômica da face, envolvendo tecidos moles da mucosa bucal, lábios, regiões oro-faringea e de língua, e comprometimentos de cordas vocais. Outras alterações sugestivas de edemas de faces são as que comprometem as de oxigenação e circulação, evidenciadas através de sinais e sintomas de taquipnéia, respiração curta e bucal, edema de mucosa bucal e de faringe, ou ainda confusão mental, hipoxia ou da presença de entubação oro-naso-traqueal fatores estes intervenientes tanto na emissão como no processamento da comunicação oral.

Outras circunstâncias do acidente do paciente queimado podem predispor diferentes causas que alteram sua comunicação, tais como: barreiras psicológicas, ausência de pessoas significativas, alteração na auto-estima e no auto-conceito. É comum observar expressões de sentimentos de raiva, culpa, ressentimento, dúvida, desespero, hostilidade, verbalizados ou não, principalmente em pacientes com 
história de tentativa de suicídio.

Assim, evidencia-se a importância da obtenção destes dados envolvidos na emissão, recepção e processamento da mensagem, para avaliação global da capacidade de comunicação do paciente queimado.

Atualmente na enfermagem, dispomos de um guia, que favorece a identificação de alteração nesta área. Trata-se da Taxonomia I da North American Nursing

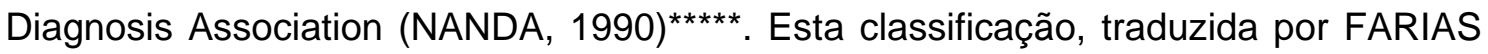
et. al. (1990), prevê o diagnóstico de enfermagem de Comunicação Verbal Prejudicada, que se encontra no anexo I.

Recentemente este diagnóstico mereceu uma proposta de ampliação (CARVALHO \& COLER, 1994), tal como se segue: Comunicação Prejudicada é o estado em que o indivíduo experimenta impedimento, diminuição ou ausência na habilidade de receber, processar, transmitir e usar um sistema de símbolos. Tem como Características Definidoras: incapacidade de falar a língua dominante; dificuldade de acompanhar e manter padrão usual de comunicação; obstinação em não falar; desorientação auto/alopsíquica; dispnéia; dificuldade de verbalizar ou falar expressa por: afonia, disfonia, distúrbio de ritmo, dislalia ou disartria; dificuldade em formar palavras ou sentenças; verbalização inapropriada; dificuldade de expressar o pensamento manifestado por: afasia, disfasia, apraxia ou dislexia; déficit auditivo parcial ou total; déficit visual parcial ou total; expressão facial/corporal de tensão; expressões de ansiedade/depressão; ausência de contato olho a olho; sentimento verbalizado e fascies inexpressivas. E como Fatores Relacionados: diferenças culturais; diferenças relacionadas ao desenvolvimento e idade; barreiras psicológicas; ações medicamentosas; barreiras ambientais; ausência de pessoas significativas; percepção alterada; déficit de informação; stress; alteração de auto-estima/auto conceito; condições físicas: barreiras físicas - traqueostomia/entubação, alteração anatômica (aparelho auditivo, visual ou fonador), alteração do sistema nervoso central, alteração do sistema neuromuscular e enfraquecimento músculo-esquelético. Como se observa esta proposta é mais ampla que a da NANDA (1990) por considerar o processo da comunicação na sua totalidade.

Em sua pesquisa inicial, através da coleta de dados empregada na assistência de enfermagem sistematizada a pacientes que sofreram queimaduras externas, DALRI (1993) encontrou, em 17 pacientes, apenas 03 com o diagnóstico de enfermagem de Comunicação Verbal Prejudicada, considerando o preconizado na Taxonomia I da NANDA (1990). Dadas as características dessa clientela, os presentes autores esperavam maior incidência deste diagnóstico de Enfermagem.

***** Esta taxonomia traz uma relação hierarquizada dos diagnósticos de enfermagem. Cada diagnóstico e composto por título, definição, características definidoras e fatores relacionados. Sugerimos como leitura complementar destes conceitos, dentre as publicações existentes, o livro difundido em nosso meio por Farias e colaboradores (1990), que trata de uma tradução pioneira da Taxonomia da NANDA 
Com a proposta de CARVALHO \& COLER (1994), que propõe uma ampliação e reformulação dos elementos constitutivos do diagnóstico de Comunicação Prejudicada, inferimos que uma análise secundária, dos dados já coletados por DALRI (1993), permitiria obter maior freqüência deste diagnóstico, bem como contribuir com a literatura nesta área, escassa. Destacamos que, no que se refere ao diagnóstico de comunicação alterada a pacientes queimados, não observamos outro estudo além do de DALRI (1993).

Assim, nossos objetivos foram:

1) verificar a incidência das características definidoras e dos fatores relacionados do diagnóstico de Comunicação Prejudicada, em pacientes internados para tratamento de queimaduras;

2) verificar a incidência da categoria diagnóstica de comunicação prejudicada nesta clientela.

\section{METODOLOGIA}

Os dados aqui apresentados são oriundos de análise secundária, conforme

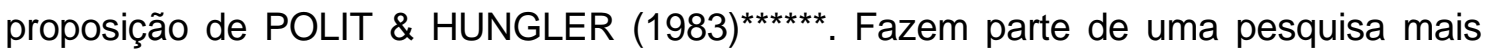
abrangente realizada por DALRI (1993). Em sua dissertação de mestrado a autora considerou como sujeitos 17 pacientes internados na Unidade de Queimaduras do HCFMRP, no período de janeiro a junho de 1992, com idade superior a 16 anos, e que apresentassem internação até 72 horas após o acidente térmico, independendo sexo, causa do acidente e agente térmico. A partir da internação a autora iniciava a coleta de dados, segundo o roteiro estabelecido (Anexo 2), elaborado de acordo com os pressupostos teóricos de HORTA (1979). Cumpre destacar que a unidade de internação adotava este instrumento para execução do Processo de Enfermagem que desenvolvia.

Julgamos oportuno salientar que a coleta de dados feita por DALRI (1993) variou de 20 a 40 minutos (tempo médio de 28,7 minutos), não apresentando relação com a superfície corporal queimada que variou de 10 a 76\%; a autora atribuiu números distintos de diagnósticos de Enfermagem para cada paciente, cuja freqüência variou de 7 a 20 diagnósticos.

Os dados coletados referidos foram objeto de reanálise; um dos instrumentos não constou do presente estudo, totalizando 16 formulários analisados. Para a realização da presente pesquisa foi elaborado um instrumento (check list), tendo como variáveis os mesmos fatores relacionados e as características definidoras descritos nas Tabelas 1 e 2, que retrata a proposta de CARVALHO \& COLER (1994) já descrita; neste instrumento eram assinaladas as evidências (sinais/sintomas) contidas na coleta de dados de cada sujeito, que justificassem o diagnóstico de Comunicação Prejudicada.

***** Análise secundária: é a forma de pesquisa na qual dados coletados para uma pesquisa são realizados por outros investigadores, usualmente para testar novas hipóteses (p.622) 


\section{RESULTADOS E DISCUSSÕES}

Observamos, nos dados categorizados segundo o instrumento proposto pelos presentes autores, a presença de várias características definidoras e fatores relacionados, propostos por CARVALHO \& COLER (1994), que nos deram suporte para estabelecer o diagnóstico de Comunicação Prejudicada, conforme se segue:

\section{- Características definidoras}

Apresentaremos inicialmente as informações concernentes às características definidoras (Tabela 1).

TABELA 1: DISTRIBUIÇÃO DAS CARACTERÍSTICAS DEFINIDORAS DO DIAGNÓSTICO DE ENFERMAGEM COMUNICAÇÃO ALTERADA EM PACIENTES PORTADORES DE QUEIMADURAS, RIBEIRÃO PRETO, 1994

\begin{tabular}{|c|c|c|c|c|c|c|c|c|c|c|c|c|c|c|c|c|c|}
\hline $\begin{array}{l}\text { CARACTERÍSTICAS } \\
\text { DEFINIDORAS }\end{array}$ & 01 & 02 & 03 & 04 & 05 & 06 & 07 & 08 & 09 & 10 & 11 & 12 & 13 & 14 & 15 & 16 & $\mathrm{~T}$ \\
\hline $\begin{array}{l}\text {-Incapacidade de falar a língua } \\
\text { dominante }\end{array}$ & & & & & & & & & & & & & & & & & 0 \\
\hline $\begin{array}{l}\text {-Dificuldade de acompanhar e } \\
\text { manter padrão usual de } \\
\text { comunicação }\end{array}$ & $\mathrm{x}$ & & & & $\mathrm{x}$ & & & $x$ & & & & & & & & & 03 \\
\hline -Obstinação em não falar & & & & & & & & & & & & & & & & & 0 \\
\hline -Desorientação auto/alopsíquica & & & & & & & & $\mathrm{x}$ & & & & & & & & & 01 \\
\hline -Dispnéia & $\mathrm{x}$ & $\mathrm{x}$ & $\mathrm{x}$ & $\mathrm{x}$ & & $\mathrm{x}$ & $x$ & & & & & & & & & $x$ & 07 \\
\hline $\begin{array}{l}\text { *Dificuldade de verbalizar ou falar } \\
\text { expressa por: afonia, disfonia } \\
\text { distúrbio em formar palavras ou } \\
\text { sentenças }\end{array}$ & & & & & & & & & & & & & & & & & 0 \\
\hline $\begin{array}{l}\text {-Dificuldade em formar palavras ou } \\
\text { sentenças }\end{array}$ & $\mathrm{x}$ & & & & & & & & & & & & & & & & 01 \\
\hline -Verbalização inapropriada & & & & & & & $x$ & & & & & & & & & & 01 \\
\hline $\begin{array}{l}\text {-Dificuldade de expressar o } \\
\text { pensamento manifestado por: afasia, } \\
\text { disfasias, apraxia ou dislexia }\end{array}$ & & & & & & & & & & & & & & & & & 0 \\
\hline *Déficit auditivo parcial ou total & & & & & & & & & & & & & & & & & 0 \\
\hline *Déficit visual parcial ou total & $x$ & $\mathrm{x}$ & & $\mathrm{x}$ & $x$ & & $\mathrm{x}$ & & $x$ & & & & & & & $x$ & 07 \\
\hline $\begin{array}{l}\text { *Expressão facial e corporal de } \\
\text { tensão }\end{array}$ & & & & & & & $\mathrm{x}$ & & & & & & & & & & 01 \\
\hline *Ansiedade/depressão & & $x$ & $\mathrm{x}$ & $x$ & $x$ & $x$ & $x$ & $x$ & $x$ & $x$ & $x$ & $x$ & $\mathrm{x}$ & & & $x$ & 13 \\
\hline *Ausência de contato olho a olho & & & & & & & & & $x$ & & & & & & & & 01 \\
\hline *Sentimento verbalizado & & & & & & & & & & & & & & & & & 0 \\
\hline *Fácies inexpressivas & & & & & & & & & & & & & & & & & 0 \\
\hline
\end{tabular}

- Elementos já validados pela NANDA

* Proposta de inclusão de Carvalho; Coler (1994) 
Foram encontradas as seguintes características definidoras na amostra estudada: expressões de ansiedade/depressão (81,25\%); déficit visual, parcial ou total (43,75\%); dispnéia (43,75\%); dificuldade de acompanhar e manter padrão usual de comunicação (18,75\%); desorientação auto/alopsíquica (6,25\%); dificuldade em formar palavras ou sentenças (6,25\%); verbalização inapropriada (6,25\%) e ausência de contato olho a olho $(6,25 \%)$.

Cumpre destacar que estes pacientes sofreram queimaduras, em decorrência de tentativa de suicídio, tentativa de homicídio, acidente de trabalho, acidente doméstico e acidente automobilístico. Foi observado ainda a verbalização de desejo de morte, angústia, raiva e tristeza. Os homens geralmente queixavam-se da dor e as mulheres, além disso, ressentiam-se do dano na imagem corporal.

O déficit parcial da visão, foi causado pela queimaduras de face; a aspiração de fumaça durante o incêndio, em ambiente fechado o contato com descargas elétricas, e a extensão corporal e profundidade da queimadura, desencadearam a dispnéia.

A proposta original da NANDA (1990) possibilitava identificar as seguintes características definidoras no trabalho original de DALRI (1993): dispnéia, dificuldade em formar palaVraS ou sentenças e verbalização inapropriada. A presente proposta possibilitou identificar, além destas, outras categorias: dificuldade de acompanhar e manter padrão usual de comunicação, déficit visual, expressão facial e corporal de tensão, ansiedade/depressão e ausência de contato olho a olho. Sete categorias não foram observadas nos pacientes estudados. Estes achados estão reiterados pelos autores anteriormente mencionados.

Cada uma destas alterações evidenciou contribuição na diminuição a habilidade de receber, processar e usar o sistema de símbolos, requerendo atenção especial do profissional enfermeiro para tais evidências.

\section{- Fatores Relacionados}

Passaremos ao exame dos fatores relacionados identificados como mostra a Tabela 2.

Obtivemos os seguintes resultados na amostra estudada: condições físicas que totalizaram $(81,25 \%)$ sendo barreira física $(6,25 \%)$ e alteração anatômica $(75,0 \%)$; stress (37,5\%); percepção alterada (25,0\%); ação medicamentosa (12,5\%) e alteração da auto-estima/auto-conceito (12,5\%).

A maior incidência recaiu na subcategoria alteração anatômica, isto é, que tiveram lesões faciais e de orofaringe por queimaduras de segundo e terceiro graus e/ou por aspiração de fumaça, apresentando edema local em intensidades variadas.

O diagnóstico da NANDA (1990) de comunicação verbal prejudicada possibilitava a DALRI (1993), para os pacientes estudados, identificar, como fatores relacionados, apenas os de barreiras físicas e alterações anatômicas.

Cumpre relembrar que estes fatores foram agrupados como condições físicas na proposta de CARVALHO \& COLER (1994). Contudo a incidência, nas duas análises, foi bastante diferente tendo também em vista a inclusão das alterações do 
aparelho visual e auditivo.

Outros fatores observados recaíram nos aspectos emocionais. As pessoas queimadas sofreram um dano, que alterará o curso de sua vida, agravados pela dor e pelo isolamento. A situação estressante, do ponto de vista físico e emocional, pode também levar a conseqüências sociais, para cada um. Havia pacientes com história de alcoolismo ou dificuldade em lidar com seus problemas e emoções, fazendo supor que tivessem traumas emocionais anteriores o que poderia causar alteração na capacidade de perceber. Tais problemas causam dificuldades na capacidade de recepção, emissão e processamento de mensagens, assim como os efeitos das ações medicamentosas, não previstas na proposta original da NANDA (1990).

TABELA 2: DISTRIBUIÇÃO DOS FATORES RELACIONADOS DO DIAGNÓSTICO DE ENFERMAGEM DE COMUNICAÇÃO ALTERADA EM PACIENTES PORTADORES DE QUEIMADURAS. RIBEIRÃO PRETO, 1994.

\begin{tabular}{|c|c|c|c|c|c|c|c|c|c|c|c|c|c|c|c|c|c|}
\hline $\begin{array}{l}\text { CARACTERÍSTICAS } \\
\text { DEFINIDORAS }\end{array}$ & 01 & 02 & 03 & 04 & 05 & 06 & 07 & 08 & 09 & 10 & 11 & 12 & 13 & 14 & 15 & 16 & $\mathrm{~T}$ \\
\hline - Diferenças culturais & & & & & & & & & & & & & & & & & 0 \\
\hline $\begin{array}{l}\text { - Diferenças relacionadas ao } \\
\text { desenvolvimento e idade }\end{array}$ & & & & & & & & & & & & & & & & & 0 \\
\hline - Barreiras psicológicas & & & & & & & & & & & & & & & & & 0 \\
\hline * Ação medicamentosa & & & & & $x$ & & & $x$ & & & & & & & & & 02 \\
\hline * Barreiras ambientais & & & & & & & & & & & & & & & & & 0 \\
\hline * Ausência de pessoas significativas & & & & & & & & & & & & & & & & & 0 \\
\hline * Percepção alterada & & & & & & & & $x$ & $x$ & & $\mathrm{x}$ & & $x$ & & & & 04 \\
\hline * Déficit de informação & & & & & & & & & & & & & & & & & 0 \\
\hline * Stress & & $x$ & & $x$ & & & $x$ & & & $x$ & & $x$ & & & & & 06 \\
\hline $\begin{array}{l}\text { * Alteração da auto-estima/auto } \\
\text { conceito }\end{array}$ & & & $\mathrm{x}$ & & & $x$ & & & & & & & & & & & 02 \\
\hline $\begin{array}{l}\text { * Condições físicas: -Barreira física- } \\
\text { traqueostomia /entubação }\end{array}$ & $x$ & & & & & & & & & & & & & & & & 01 \\
\hline $\begin{array}{l}\text { - Alteração anatômica (aparelho } \\
\text { visual, fonador e auditivo) }\end{array}$ & $x$ & $x$ & $x$ & $x$ & $x$ & $x$ & $x$ & $x$ & $x$ & & $x$ & & $x$ & & & $x$ & 12 \\
\hline - Alteração do SNC & & & & & & & & & & & & & & & & & 0 \\
\hline $\begin{array}{l}\text { - Alteração no sistema } \\
\text { neuromuscular }\end{array}$ & & & & & & & & & & & & & & & & & 0 \\
\hline $\begin{array}{l}\text { - Enfraquecimento músculo- } \\
\text { esquelético }\end{array}$ & & & & & & & & & & & & & & & & & 0 \\
\hline
\end{tabular}

- Elementos já validados pela NANDA

* Proposta de inclusão de Carvalho; Coler (1994)

Rev. Latino-am.enfermagem - Ribeirão Preto - v. 4 - n. especial - p. 153-172 - abril 1996159 


\section{- Categoria Diagnóstica}

Como já mencionado, nos dados de DALRI (1993), observamos que dos 17 pacientes da análise primária pela Taxonomia I Revisada da NANDA ano (1990), apenas 03 deles $(17,6 \%)$ apresentaram o diagnóstico de Comunicação Prejudicada. Em análise utilizando a proposta de CARVALHO \& COLER (1994), obtivemos 14 diagnósticos de Comunicação Prejudicada dos 16 pacientes analisados (87,5\%).

Evidenciamos, com tal resultado, que a proposta de CARVALHO \& COLER (1994) demonstrou maior sensibilidade para a avaliação da Comunicação Prejudicada, na amostra selecionada, com o mesmo banco de dados dos sujeitos observados.

\section{- Considerações Gerais}

Inicialmente destacamos as interfaces existentes entre os diversos padrões de respostas humanas propostos pela NANDA (1990) e de seus diagnósticos de enfermagem, bem como as variações de incidência de um perfil diagnóstico de uma clientela em decorrência da amplitude das definições dos diagnósticos considerados.

Observamos algumas dificuldades com a referida proposta, como a de interpretação do fator, relacionado "percepção alterada"; sugerimos a exemplificação ou elaboração de definição para este fator com vistas a minimizar erros de categorização. Outro aspecto diz respeito ao fator relacionado "stress", e a sua proximidade com as "expressões de ansiedade/depressão" mencionadas como uma característica definidora. Embora se compreenda a relação cíclica entre comunicaçãostress-ansiedade/depressão, estudos específicos para determinação desses elementos (como fatores relacionados ou características definidoras) necessitam ser desenvolvidos. Outro item de interpretação difícil, diz respeito à característica definidora, "sentimento verbalizado". Pode haver dupla interpretação onde o por um lado, o paciente relate os seus sentimentos (raiva, tristeza, culpa) e, por outro, onde o paciente refira percepção de Comunicação Prejudicada.

Atentamos também para a necessidade de ampliar o conhecimento dos profissionais sobre os fatores relacionados e as características definidoras do diagnóstico de enfermagem de Comunicação Prejudicada, no que se refere às suas particularidades, para que não ocorram erros por omissão ou execução.

Merece ainda destaque a escassa referência bibliográfica sobre avaliação das alterações da comunicação, sendo quase inexistente, em nosso meio, as que abordam as lesões por queimaduras.

\section{CONSIDERAÇÕES FINAIS}

Julgamos necessário reiterar que, para se estabelecer um diagnóstico de enfermagem é necessário identificar com precisão os sinais e sintomas, através de 
dados significativos obtidos na coleta de dados, bem como, analisá-los de forma global e não apenas sob a ótica de um problema específico. Assim, é imprescindível avaliar todos os dados significativos coletados de pacientes portadores de queimaduras, ao se buscar a identificação completa e correta dos fatores relacionados e das características definidoras do diagnóstico de enfermagem de Comunicação Prejudicada. Finalmente, é imperioso considerar as múltiplas facetas que podem estar envolvidas no processo comunicacional, inclusive as decorrentes de situações como queimaduras. Esta adoção, por parte da NANDA ano (1990), permitirá retratar as alterações de comunicação em suas múltiplas ocorrências.

\section{IMPAIRED COMMUNICATION, RELATED FACTORS AND DEFINING CHARACTERISTICS IN BURNED PATIENTS}

The present work studies questions related to burned patients and their influences in the communication process. It aims at contributing to the nursing concern about the creation of tools to identify communication problems, to formulate nursing diagnoses involving these changes as well as to elaborate alternative proposals in the care of patients with impaired communication. The results found by DALRI (1993) showed 3 patients with the diagnosis of impaired communication. The authors, utilizing the Taxonomy I of the North American Nursing Diagnosis Association (NANDA) 1990, analyzed the data using the proposal of CARVALHO \& COLER (1994). They found 14 patients with this alteration (Impaired Communication), confirming the hypothesis that the second proposal for the diagnoses of Impaired Communication is more comprehensive. Frequent related factors were: physical conditions - anatomical alterations (visual and phono system); stress and altered perception. Common defining characteristics were: expressions of anxiety/depression; partial and total visual deficit and dyspneia.

UNITERMS: nursing diagnosis; impaired communication

\section{COMUNICACIÓN ALTERADA, FACTORES RELACIONADOS Y CARACTERÍSTICAS QUE LA DEFINEN, EN PACIENTES INTERNADOS CON QUEMADURAS}

El presente estudio aborda las cuestiones del paciente portador de quemaduras y sus influencias en el proceso de comunicación. Busca contribuir con la preocupación reciente de la Enfermería con la creación de instrumentos de identificación de problemas 
de comunicación, la formulación de diagnósticos de enfermería que envuelven estas alteraciones y también, la elaboración de propuestas alternativas para el cuidado de pacientes con comunicación alterada. A partir del conocimiento de los resultados de DALRI (1993), quien encontró 03 pacientes con diagnósticos de comunicación alterada, utilizando la Taxonomía I de la North American Nursing Diagnosis Association (NANDA) 1990 ano las autoras realizaron un análisis secundário de los datos, tomando como ayuda la propuesta de CARVALHO \& COLER (1994). En esta muestra, encontraron 14 pacientes con esta alteración (Comunicación Alterada), confirmando la hipótesis de que esta segunda propuesta para el diagnóstico de enfermería de Comunicación Alterada, presentase más comprensiva. Los factores relacionados más frecuentes fueron: condiciones físicas- alteración anatómica (aparato visual y fonador): stress y percepción alterada. Las características definidoras mas comunes fueron: expresiones de ansiedad/depresión; déficit visual parcial o total y disnéa.

UNITERMOS: diagnóstico de enfermería; comunicación alterada

\section{REFERÊNCIAS BIBLIOGRÁFICAS}

01. ARTZ, C.P.; MONCRIEF, J.A.; PRUIT-Jr, B.A. Queimaduras. Rio de Janeiro:, Interamericana, 1980.

02. CARVALHO, E.C.; COLER, M.S. Diagnosis of human response pattern: communicating /Apresentado no NANDA - $11^{\text {th }}$ bienal conference on nursing diagnosis. Março, 1994 - Nashville - TN - USA./ (mimeografado)

03. DALRI, M.C.B. Perfil diagnóstico de pacientes queimados segundo o modelo conceitual de Horta e a taxonomia I revisada da NANDA. Ribeirão Preto, 1993. 203p. Dissertação (Mestrado) - Escola de Enfermagem de Ribeirão Preto, Universidade de São Paulo.

04. FARIAS, J.N. et al. Diagnóstico de enfermagem: uma abordagem conceitual e prática. João Pessoa: Santa Marta, 1990.

05. GOMES, D.R.; SERRA, M.C.; PELLON, M.A. Queimaduras. Rio de Janeiro: Revinter, 1995.

06. HORTA, W.A. Processo de enfermagem. São Paulo: EPU/EDUSP, 1979.

07. LYNCH, J. B.; LEWIS, S.R. (eds.) Symposium on treatment of burns. In: SYMPOSIUM OF THE EDUCATIONAL FOUNDATION OF THE AMERICAN SOCIETY OF PLASTIC AND RECONSTRUCTIVE SURGEONS, Texas, 1971.

Procedings. Saint Louis, C.V. Mosby, 1973. v.5

08. McFARLAND, G.K.; NASCHINSKI, C.E. Impaired communication e descriptive study. Nurs. Clin. North América, v. 22, n. 4, p. 775-785, 1985. 
09. NANDA - North American Nursing Diagnosis Association. Taxonomy I - St. Louis, 1990.

10. POLIT. D.; HUNGLER, B. Nursing research - principles and methods. 2. ed. Philadelphia, J. B. Lippincott, 1983.

11. SMELTZER, S.C.; BARE, B. G.; Brunner/Suddarth tratado de enfermagem medico-cirúrgico. 7 ed. Rio de Janeiro: Guanabara Koogan, 1994. v. 3, cap. 52, p. $1275-305$. 


\section{ANEXO I}

\section{2 - PADRÃO DE RESPOSTA HUMANA - COMUNICAR}

\subsubsection{1- COMUNICAÇÃO VERBAL PREJUDICADA (1973)}

DEFINIÇÃO:

O estado em que o indivíduo experimenta uma diminuição ou uma ausência de habilidade para usar ou entender a linguagem na interação humana.

CARACTERÍSTICAS DEFINIDORAS:

* Incapacidade para falar a língua dominante;

* Dificuldade de verbalizar ou de falar;

* Impossibilidade de falar e obstinação em não falar;

Gagueira;

Disartria;

Dificuldade para formar palavras ou sentenças;

Dificuldade para expressar verbalmente o pensamento;

Verbalização inapropriada;

Dispnéia;

Desorientação;

\section{FATORES RELACIONADOS:}

Diminuição na circulação cerebral;

Tumor cerebral;

Barreira física (traqueostomia, entubação);

Defeito anatômico (por ex.: fenda palatina);

Barreiras psicológicas (psicose, falta de estímulo);

Diferenças culturais;

Diferenças relacionadas ao desenvolvimento ou à idade.

* Característica definidora crítica.

FONTE: Farias (1990. pág. 104, 105) 


\section{ANEXO II}

IMPRESSO $\mathrm{n}^{\circ} \mathrm{I}$ - Instrumento de Coleta de Dados

COLETA DE DADOS

$\overline{\text { DATA }}$
A -IDENTIFICAÇÃO
1 -Como você gostaria de ser chamado?
2 - Local do acidente:
3 - Ambiente ( ) aberto ( ) fechado
4 - Motivo, data e hora da queimadura

I - NECESSIDADES PSICOBIOLÓGICAS

1 - Necessidades de Oxigenação

- freqüência:

$\mathrm{mov} / \mathrm{min}$.

- ritmo: ( ) regular ( ) irregular

- expansão da caixa torácica

( ) simétrica ( ) assimétrica

( ) profundo ( ) superficial

- outras alterações:

- presença de secreções ( ) sim （）não

características:

localização:

Fonte: Dalri (1993, p. 27 a 32)

Rev. Latino-am.enfermagem - Ribeirão Preto - v. 4 - n. especial - p. 153-172 - abril 1996165 
- queimadura de face ( ) sim ( ) não

profundidade:

extensão:

- queimaduras de vias aéreas ( ) sim ( ) não

extensão:

- queimaduras de vibriças ( ) sim ( ) não

- aspiração de fumaça ( ) sim ( ) não

- presença de queimadura na região tóraco-cervical que possa comprometer a respiração?

( ) $\operatorname{sim}($ ) não

extensão:

- outras alterações:

2 - Necessidade de Circulação

- freqüência cardíaca: $\mathrm{bat} / \mathrm{min}$.

( ) rítmica ( ) arrítmica

- características do pulso:

( ) cheio ( ) fraco ( ) forte

outras alterações:

- pressão arterial $\mathrm{mmHg}$

características do som:

posição do paciente:

locais para verificação:

- edema ( ) sim ( ) não

intensidade:

localização:

- áreas com queimaduras circunferencial de $3^{\circ} \mathrm{Grau}$

( ) $\operatorname{sim}($ ) não

localização:

perfusão:

Outras alterações: 
- rede vascular:

locais não queimados para punção venosa:

arterial:

Outras alterações:

3 - Necessidade de Termorregulação

$\mathrm{T}=$ ${ }^{\circ} \mathrm{C}$

Outras alterações:

4 - Necessidade de Integridade Tecidual

- região queimada:

- cicatrizes ( ) sim ( ) não

região:

especificar:

- lesões não decorrentes das queimaduras ( ) sim ( ) não

região:

características:

- presença de pêlos ( ) sim ( ) não

locais:

- Outras alterações:

5 - Necessidade de Percepção Sensorial

- dor ( ) sim ( ) não

experiências anteriores com dor:

no momento sente ( ) dor ( ) desconforto

localização:

características da dor:

está associada a:

( ) posição ( ) exercícios ( ) alimentação

( ) ansiedade ( ) terapia ( ) queimaduras de $1^{\circ}$ e $2^{\circ}$ Graus

- nível de consciência ( ) sim ( ) não

abertura ocular

( ) espontânea ( ) ordem verbal ( ) dor ( ) nenhuma

resposta motora:

( ) obedece a comandos

( ) flexão normal a dor

( ) localiza a dor

( ) extensão a dor

( ) nenhuma

Outras alterações: 
- acuidade visual:

capacidade de ver objetos ou pessoas:

( ) próximos ( ) distantes

capacidade de ler:

( ) $\operatorname{sim}$ ( ) não

uso de:

( ) óculos ( ) lentes ( ) próteses

Outras alterações:

- acuidade olfativa:

capacidade de discriminar odores: ( ) sim （ ) não

hipersensibilidade:

( ) $\operatorname{sim}$ ( ) não

Outras alterações:

- acuidade auditiva:

capacidade de distinguir sons: ( ) sim ( ) não

Outras alterações:

- gustativa:

capacidade para distinguir sabores:

( ) $\operatorname{sim}$

( ) não

Outras alterações:

- tato:

capacidade de sentir a natureza e forma dos objetos

( ) $\operatorname{sim}$ ( ) não

Outras alterações:

6 - Necessidade de Alimentação e Hidratação

- alimentação

peso: $\quad \mathrm{Kg}$ altura $\_\mathrm{m}$

estado nutricional:

tipos de alimentos que costuma ingerir:

quantidade:

horário:

dietas especiais:

- hidratação:

turgor da pele: ( ) pastoso ( ) semi-pastoso

( ) fino ( ) frouxo

Rev. Latino-am.enfermagem - Ribeirão Preto - v. 4 - n. especial - p. 153-172 - abril 1996168 
tipos de líquidos que costuma ingerir:

quantidade:

horário:

7 - Necessidade de Eliminação

- fezes:

freqüência:

( ) constipação ( ) diarréia ( ) incontinência

características:

Outras alterações:

- urina:

freqüência:

( ) retenção urinária ( ) incontinência

quantidade:

características:

odor:

Outras alterações:

8 - Necessidades de Sono e Repouso

- hábitos:

- dificuldade:

- uso de medicação:

- Outras alterações:

9 - Necessidade de Atividade Física

- atividade física:

- locomoção:

- dificuldades:

- musculatura:

( ) força presente ( ) paresia

( ) parestesia ( ) plegias

( ) uso de próteses ( ) deformidades

( ) lesões

- Outras alterações:

- queimaduras nas articulações: ( ) sim ( ) não

extensão:

profundidade:

localização:

- queimadura nos pés: ( ) sim ( ) não .

extensão:

profundidade: 
10 - Necessidade de Higiene

- higiene corporal:

freqüência:

tipo de banho:

- higiene oral:

freqüência:

presença de próteses: ( ) sim ( ) não

quais:

ausência de dentes:

quais:

estado dos dentes:

- cuidado com os cabelos:

11 - Necessidade de Abrigo

- condições de habitação:

tipo de material de construção:

( ) alvenaria ( ) madeira ( ) barro

número de cômodos:

número de pessoas na casa:

- saneamento básico da casa:

( ) luz elétrica ( ) lamparina

( ) água encanada ( ) poço

( ) rede de esgoto ( ) fossa negra ( ) fossa seca

( ) coleta de lixo-destino:

distância entre o poço e a fossa:

- Outras alterações:

12 - Necessidade de Integridade Física

- vícios e hábitos

( ) uso de drogas ( ) auto medicação

( ) tabagismo ( ) alcoolismo

Outros:

- realiza exames periódicos: ( ) sim ( ) não

qual:

- vacinação:

- tem ou teve algum problema de saúde?

( ) $\operatorname{sim}$ ( ) não

Qual: 
13 - Necessidade de Sexualidade

- mantém algum relacionamento sexual?

- sente que sua doença poderá interferir na sua atividade sexual?

- Outras alterações:

- queimaduras dos órgãos genitais: ( ) sim （ ）não

extensão:

- dia da última menstruação:

- Outras alterações (gestações, abortos e amamentação):

\section{II - NECESSIDADES PSICOSSOCIAIS}

1 - Necessidade de Segurança

( ) ansiedade ( ) medo ( ) agressividade ( ) aflições

( ) calmo ( ) retraído ( ) incapacidade ( ) desamparo

( ) potencial para violência

reações ao trauma e stress:

- Outras alterações:

2 - Necessidade de Comunicação:

- distúrbio de fala:
( ) afasia
( ) dislalia
( ) falante
( ) silêncio

( ) monotonia ( ) exuberância ( ) reticente

- Outras alterações:

- distúrbio de linguagem:
( ) clareza
( ) coerência

( ) respostas desconexas

- Outras alterações:

3 - Necessidade de Interação Social

( ) permanece sozinho

( ) comunica-se com outros

( ) participa de atividades

( ) provoca situações de descontentamento

- gostaria de receber visitas durante a internação:

( ) sim ( ) não

De quem: 
4 - Necessidade de Lazer e Recreação

O que gostaria de fazer durante a internação para ocupar-se?

5 - Necessidade de Auto-Estima

O que sente sobre trauma causado pela queimadura?

6 - Necessidade de Auto-Realização

Como pensa que a queimadura poderá afetar a sua vida?

- expectativa de vida:

III - NECESSIDADES PSICOESPIRITUAIS

- Crença religiosa:

- Gostaria de praticar alguma atividade religiosa durante a internação?

B - OUTRAS INFORMAÇÕES IMPORTANTES PARA A ENFERMAGEM

Assinatura do Enfermeiro

Data: / /

COREn: 